Pacific Journal of Mathematics

CONSTRUCTION OF IRREDUCIBLE HOMOLOGY 3-SPHERES 


\title{
CONSTRUCTION OF IRREDUCIBLE HOMOLOGY 3-SPHERES WITH ORIENTATION REVERSING INVOLUTION
}

\author{
L. C. Siebenmann and J. M. Van Buskirk
}

A simple procedure is described for constructing infinitely many homology 3-spheres which admit an orientation reversing involution with two fixed points, yet are irreducible (in that each smoothly embedded 2-sphere bounds a 3-ball); they are 2-fold branched cyclic coverings of suitably chosen knots.

1. Introduction. It is appropriate to explain why Ron Stern asked us if examples such as those herein exhibited could be found. They make it seem a little more difficult to triangulate all closed topological manifolds of dimension $\geq 5$ so as to make them finite simplicial complexes. Recall first that the triangulation theory of D. E. Galewski and R. J. Stern or of T. Matumoto, combined with the double suspension theorem $\left(\Sigma^{2} H^{n} \approx S^{n+2}, n \geq 3\right.$ ) of R. D. Edwards and J. W. Cannon show that such a triangulation is possible if and only if there exists a smooth closed homology 3-sphere $H^{3}$ satisfying

(a) the Rohlin invariant $\mu\left(\mathrm{H}^{3}\right)$ in $\mathbf{Z} / 2$ of $\mathrm{H}^{3}$ is non-zero, and

(b) $\mathrm{H}^{3} \# \mathrm{H}^{3}$ bounds a smooth acyclic 4-manifold.

Appropriate references appear in [9].

Now (b) is certainly verified if there is an orientation reversing diffeomorphism of $H^{3}$, for then

$$
H^{3} \#\left(-H^{3}\right)=\partial\left(H_{0}^{3} \times[0,1]\right),
$$

where $H_{0}^{3}$ is $H^{3}$ minus the interior of a 3-ball. Reasonably enough, topologists began by testing $H^{3}$ 's that admit an orientation reversing involution; however J. S. Birman [3], Galewski and Stern [9], and W. C. Hsiang and P. Pao [12] have recently proved (in three different ways) that any homology 3-sphere with orientation reversing involution has Rohlin invariant zero.

We are providing non-trivial examples to which this vanishing theorem applies, in contrast to the hitherto known examples which were derived from $S^{3}$ with an orthogonal orientation reversing involution by (equivariant) connected sum, namely $H^{3} \# S^{3} \#\left(-H^{3}\right)$ (the summation being taken away from the fixed points). Indeed, it was believed, for a 
short time, that any homology 3-sphere admitting an orientation reversing involution was necessarily of the form $H^{3} \#-H^{3}$ (from which the vanishing of the Rohlin invariant follows trivially).

We restate our result:

THEOREM. There exist infinitely many irreducible homology 3-spheres with orientation reversing involution fixing two points.

The examples produced are 2-fold coverings $M_{K}^{3}$ of $S^{3}$ branched over knots $K$ carefully constructed (in $\$ 2$ ) to satisfy

(1) the determinant, $\delta_{K}$ of $K$ is 1 (see $\S 4$ ).

(2) $K$ is strongly positive amphicheiral (see §2).

(3) $K$ is prime.

The determinant condition (1) implies that $M_{K}$ is a homology 3-sphere (§3).

Condition (2) assures the existence of an orientation reversing involution ( $\$ 2)$.

The irreducibility of $M_{K}$ follows from condition (3), since a necessary and sufficient condition for the irreducibility of such branched covers is that $K$ be prime (necessity [23]; sufficiency [14]). Actually, we will show directly (\$5) that (3') $M_{K}$ is irreducible.

Joan Birman has indicated to us that other examples can be given using Heegard diagrams as in [3] together with W. Haken's (easy!) theorem [11] to the effect that any Heegard splitting of a connected sum $M_{1}^{3} \# M_{2}^{3}$ is a sum of Heegard splittings of $M_{1}^{3}$ and $M_{2}^{3}$.

We would like to thank Ron Stern for encouragement, John Conway for advertising his efficient knot polynomial methods, and Ray Lickorish for organizing a very congenial 1978 summer seminar at Cambridge University around which all this transpired.

2. The construction. A knot is strongly positive amphicheiral if, as the 3-strand, 5-bight Turk's head knot in Figure 1(a), it is invariant under reflection $\alpha$ through the origin 0 , but it does not pass through the origin so that its string orientation is necessarily preserved by $\alpha$. Beware that there are other forms of rigid positive amphicheirality; namely, by rotary reflection of order $4,8,16, \ldots, 2^{k}, \ldots$ Here $\alpha$ is a rotary reflection of order 2 (cf. [5] and [22]).

Now if $\tilde{\alpha}$ is either of the two automorphisms of $M_{K}$ covering $\alpha$, then, since $\tilde{\alpha}^{2}$ covers the identity, we see that $\tilde{\alpha}^{2}$ is either the identity or the covering translation. It is not the latter, since $\tilde{\alpha}^{2}$ fixes the $t w o$ points in $M_{K}$ 
over the origin; so we conclude that the orientation reversing diffeomorphism $\tilde{\alpha}$ has period 2, and hence that for any strongly positive amphicheiral $k$ kot $K$, the covering $M_{K}$ has an orientation reversing involution. We note that $\tilde{\alpha}$ would have had period 4 if $K$ had passed through the origin (and hence through $\infty$ ).

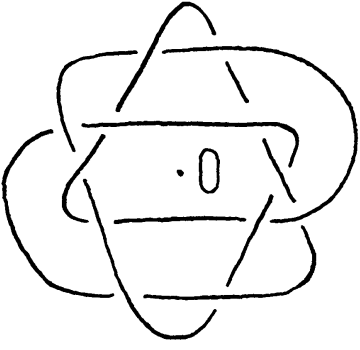

(a)

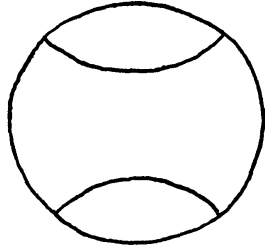

(b)

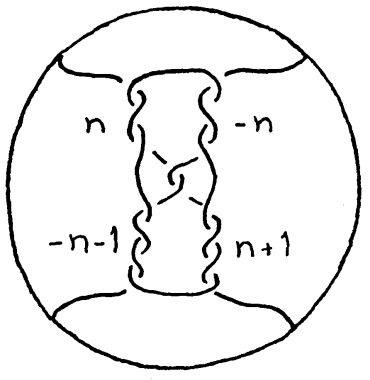

(c)

FIGURE 1

Our description of knots satisfying properties (1), (2) and (3') will employ tangles in the sense of J. H. Conway [6]. A tangle $T$ consists of a copy of a standard 3-ball containing a given smooth 1-submanifold (also called $T$ ) which meets the boundary transversely in four fixed standard points, say $( \pm \sqrt{2}, \pm \sqrt{2}, 0)$, of an equatorial circle as in Figure $1(b)$. Our tangles are, for convenience, somewhat special in that (see Figure 2(a))

(i) $T$ joins points of the equatorial circle which are horizontally, or vertically, opposite one another (rather than joining diagonally opposite points).

(ii) $T$ carries an orientation of its strings so that the entry and exit points alternate as the equatorial circle is traversed.

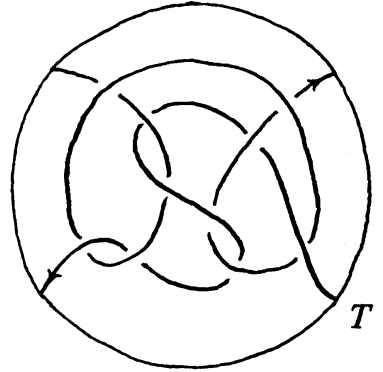

(a)

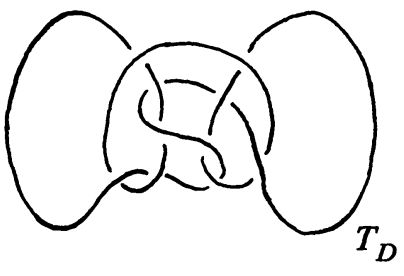

(b)

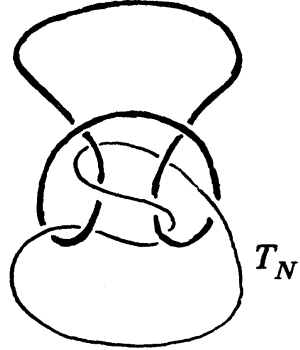

(c)

Figure 2 
In Figures 2(b) and (c) the tangle $T$ of Figure 2(a) is completed to oriented 1-submanifolds of $S^{3}$ called respectively the denominator $T_{D}$ and the numerator $T_{N}$ of $T$, one of which is a knot and the other a link of two components. Observe that, by (ii), $T_{N}$ and $T_{D}$ have unique orientations compatible with the orientations of $T$. When $T$ has two component strings (as all of our examples will) there are just two orientations (of its strings) permitted by (ii).

Our construction of knots satisfying (1), (2) and (3') is indicated in Figure 3(a), where the copies of the tangle $P$ in the two outermost positions are reflections of one another in the origin 0 , as is also the case for the copies of $Q$ in the innermost positions.

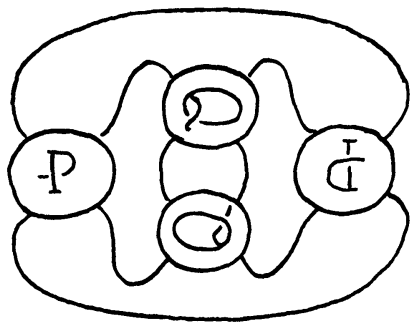

(a)

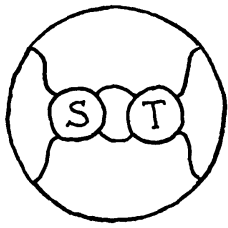

(b)

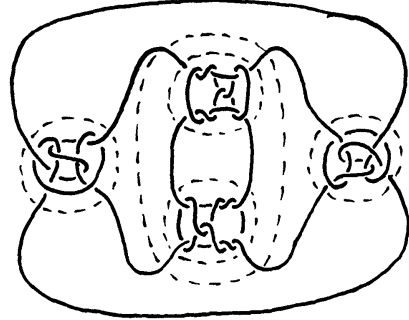

(c)

FIGURE 3

We choose the tangles $P$ and $Q$ (which have knots as denominators) such that

$(\alpha)$ the 2-fold branched covers of $S^{3}$ over the denominators $P_{D}$ and $Q_{D}$ of the tangles $P$ and $Q$ are homology 3-spheres; equivalently, the denominators have determinant \pm 1 [19], [20, p. 213]

( $\beta$ ) the 2 -fold branched covers $\tilde{B}_{P}$ and $\tilde{B}_{Q}$ of the containing 3-balls branched over the tangles $P$ and $Q$ are irreducible; sufficiently, the numerators of $P$ and $Q$ are unlinks (cf. Lemma (A) in §5)

$(\gamma)$ neither $\tilde{B}_{P}$ nor $\tilde{B}_{Q}$ is a solid torus; equivalently, neither $P$ nor $Q$ is (diffeomorphic to) the null tangle of Figure 1(b) (cf. Lemma (B) in §5).

The tangles of Figures 1(c) and 2(a), which replace the tangles $P$ and $Q$ in Figure 3(a) to form the knot of Figure 3(c), satisfy these conditions: the determinant condition $(\alpha)$ is discussed in $\$ 3$; the irreducibility and non-triviality conditions are verified in $\$ 5$.

A simpler choice for $P$ and $Q$ is the tangle of Figure 4(a); its 2-fold branched cyclic covering is the Seifert fiber space over $B^{2}$ with 3 exceptional fibers of types $1 / 2,1 / 3,1 / 5$ (cf. [18]). This is irreducible with incompressible torus boundary by [24]; thus $(\beta)$ and $(\gamma)$ are verified. The 
denominator is the $(3,5)$ torus knot with 2 -fold branched cyclic covering the Poincaré homology 3-sphere (cf. [20, p. 309]); hence $(\alpha)$ is verified. There are infinitely many similar examples whose 2 -fold branched cyclic covers are distinct Seifert fiber spaces over $B^{2}$ (cf. [21, Chap. II], [18]).

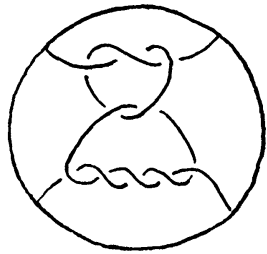

(a)

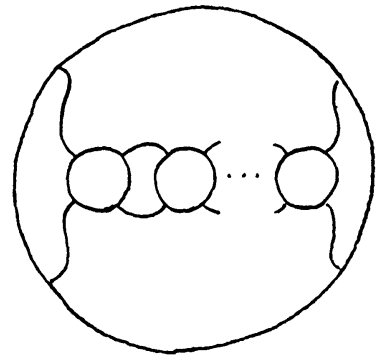

(b)

Figure 4

A simpler way to generate infinitely many examples is to add together $k=1,2,3, \ldots$ copies of the tangle $4(\mathrm{a})$, as indicated in Figure $4(\mathrm{~b})$ (condition $(\alpha)$ is clear; as for $(\beta)$ and $(\gamma)$, the 2 -fold branched coverings of the tangles are distinct irreducible graph manifolds classified by $F$. Waldhausen [24] (cf. [5])).

When $K$ is constructed from the iterated sum of tangles as in Figure 4(b), the 2-fold covering $M_{K}^{3}$ is itself an irreducible graph manifold whose compact characteristic Seifert manifold part [13] includes $2 k+2 k^{\prime}$ copies of the 2-fold cover of tangle 4(a) (supposing $P$ is a $k$-fold sum and $Q$ a $k^{\prime}$-fold sum). Thus we have infinitely many examples as our theorem asserts.

The tangles 1(c) and 2(a) have 2-fold coverings that are neither Seifert nor graph manifolds. Although the irreducibility of such manifolds is sometimes difficult to establish, ad hoc considerations, as in $§ 5$, often work. The addition trick of Figure 4(b), used to generate infinitely many examples, still works; with these tangles, the $2 k+2 k^{\prime}$ Seifert components mentioned above are replaced by at least as many atoroidal (hyperbolic) components; as for irreducibility, the reader will find that the arguments of $\S 5$ apply.

3. Recollections about determinants. We exploit Conway's methods of calculation [6]. For any link $L$ (i.e. oriented 1-submanifold of $S^{3}$ ) there is defined a genuine polynomial $P_{L}(x)$ with integer coefficients which (for a knot) is related to the usual Alexander polynomial $\Delta_{L}$ by $P_{L}\left(x-x^{-1}\right)=$ $\Delta_{L}\left(x^{2}\right)$. 
All of the properties of $P_{L}(x)$ except its existence follow (often trivially) from $P_{L}(x)=1$ for the unknot and Conway's first identity (compare Alexander's relation 12.2 [1, p. 302])

$$
P_{+}(x)=P_{-}(x)+x P_{0}(x)
$$

where $L_{+}, L_{-}$and $L_{0}$ are links that differ only in a single vignette where we see respectively
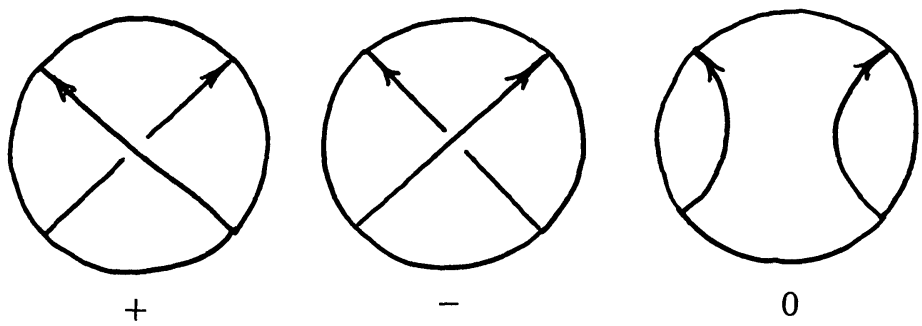

(we have written $P_{+}$for $P_{L_{+}}$, etc.).

Conway recommends that $P_{L}(x)$ be defined in the first place by using the matrix methods of J. W. Alexander [1] with suitable normalizations (cf. [7]. Conway's article [6] discusses the Laurent polynomial $D_{L}(x)=$ $P_{L}\left(x-x^{-1}\right)$ rather than $P_{L}$, which makes (*) a little more difficult to use).

For a link $L$ of an odd [even] number of components, $P_{L}(x)$ involves only even [respectively odd] powers of $x$.

The determinant $\delta_{L}$ of $L$ is defined to be $P_{L}(2 i)$ where $i^{2}=-1$. Then, for a $\operatorname{knot} \delta_{L}=\Delta_{L}(-1)$ is an integer, while for a link of 2 components it is $i$ times an integer.

We shall need to know that simultaneous reversal of all string orientations leaves $P_{L}(x)$ and $\delta_{L}$ unchanged, while reversal of ambient orientation (i.e. mirror reflection) changes $P_{L}(x)$ to $P_{L}(-x)$ and hence changes $\delta_{L}$ to its complex conjugate $\bar{\delta}_{L}$.

EXAmple. For the 2-component unlink, $P_{L}(x)=0$. For the $(2, n)$ torus link (i.e., the closure of the 2-component braid $\sigma_{1}^{n}$ ), the determinant is $n i^{n-1}$, which for $n=0,1,2, \ldots$ is $0,1,2 i,-3,-4 i, 5,6 i,-7,-8 i, \ldots$

For a knot $K$ we recall that $P_{K}\left(x-x^{-1}\right)=\Delta_{K}\left(x^{2}\right)$, where $\Delta_{K}(t)$ is the Alexander polynomial in $\mathbf{Z}\left[t, t^{-1}\right]$ normalized so that $\Delta_{K}(1)=1$ and $\Delta_{K}(t)=\Delta_{K}(-t)($ cf. [16]).

For a 2-component link $L$, the polynomial $P_{L}\left(x-x^{-1}\right)$ is, up to multiplication by an odd power of $x$ equal to $\Delta_{L}\left(x^{2}\right)$ where $\Delta_{L}(t)$ is a polynomial that Alexander defined for such links [1, p. 296]. In terms of 
the polynomial $\Delta_{L}(u, v)$ which R. H. Fox termed the Alexander polynomial of the 2-component link $L$ in $[8$, p. 131] we have

$$
P_{L}\left(x-x^{-1}\right)=\left(x-x^{-1}\right) \Delta_{L}\left(x^{2}, x^{2}\right)
$$

(which is plausible if we recall that $P_{L}(x)$ is divisable by $x$ in $\mathbf{Z}[x]$ ).

A precious tool for the calculation of determinants is the determinant fraction of an (oriented) tangle $T$; it is the formal fraction (integer pair) $\delta\left(T_{N}\right) / \delta\left(T_{D}\right)$, also written $T_{n} / T_{d}$. According to Conway [6, p. 336], the determinant fraction of the sum $S+T=U$ of (compatibly oriented) tangles $S$ and $T$, illustrated in Figure 3(b), depends only on the determinant fractions of $S$ and $T$. Its formula, which the notation anticipates, is the formal fraction sum

$$
U_{n} / U_{d}=\left[S_{n} T_{d}+T_{n} S_{d}\right] / S_{d} T_{d} .
$$

Rather than directly compute the determinants of the denominators of the tangles of Figures 2(a) and 1(c), in verifying the determinant condition $(\alpha)$ for these tangles, we will use the fact that the denominator of the former (pictured in Figure 2(b)) is a 10-crossing knot with Alexander polynomial $\Delta(t)=t^{3}-t^{2}-t+3-t^{-1}-t^{-2}+T^{-3}$ [20, p. 414], while that of the latter is, for $n \geq 2$, a non-trivial knot with trivial Alexander polynomial [15]. In each case $\delta_{K}=\Delta(-1)=1$.

4. $M_{K}^{3}$ is a homology 3-sphere. It suffices to show that the determinant of any knot $K$ constructed in $\S 2$ is \pm 1 , and for this calculation we exploit Conway's determinant fraction of a tangle introduced in $\$ 3$.

Consider the construction of $K$ illustrated in Figures 3(a) and (c). Note that if $P$ has determinant fraction $P_{n} / P_{d}$, the reflection of $P$ in the origin (of Figure 3(a)) has fraction $\left(-P_{n}\right) / P_{d}$. Also, $P$ rotated $\pi / 2$ in the plane has fraction $P_{d} / P_{n}$. Thus we calculate that the two composite tangles that face each other across the dotted ellipse of Figure 3(c) have determinant fractions $P_{n} / P_{d}+\left(-P_{n}\right) / P_{d}=0 / P_{d}^{2}$ and $Q_{d}^{2} / 0$ and that their sum has determinant fraction $\left(P_{d} Q_{d}\right)^{2} / 0$. (In fact, the determinant of any strongly positive amphicheiral knot is a square [22], while that of any positive amphicheiral link of 2-components is 0 [6, p. 340]). Since $K$ is the numerator of the sum of these two tangles, it follows that its determinant $\delta_{K}$ is $( \pm 1 \cdot \pm 1)^{2}=1$.

5. Irreducibility of $M_{K}^{3}$. This remains to be established when $K$ is built, as in Figure 3(c), using the tangles of Figure 1(c) or 2(a). We shall show that $M_{K}^{3}$ is the result of fitting six irreducible 3-manifolds together 
along their incompressible boundaries; namely, along the four tori covering the 2-sphere boundaries of the 3-balls containing the prime tangles in $K$ and the torus covering a 2-sphere separating the central pair of tangles from the others. The position of these five 2-spheres is indicated in Figure 3 (c) for a specific knot $K$. It then follows classically that $M_{K}^{3}$ is irreducible (since a 2-sphere in $M_{K}^{3}$, which has been isotoped to have minimal transversal intersection with the five tori, could have on it no "innermost" component of the intersection, it would not meet the tori).

First consider the two pieces of $M_{K}^{3}$ (of the six defined above) which have three boundary components. They are identical, each being the 2-fold covering of a 3-ball with two 3-balls removed from its interior, branched over six arcs situated, as in Figure 5(a), so that each pair of its boundary components is joined by a pair of arcs. Since this branched covering is $S^{1} \times D_{00}$, where $D_{00}$ is a disc with two discs removed from its interior, shown in Figure 5(b), it is irreducible with incompressible boundary.

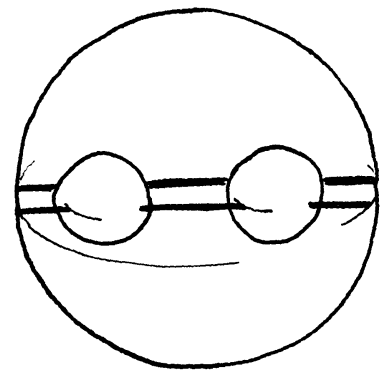

(a)

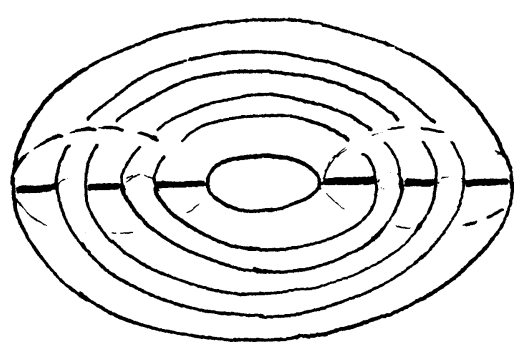

(b)

FIGURE 5

Next consider the four pieces of $M_{K}^{3}$ which are 2-fold coverings $\tilde{B}_{A}^{3}$ of $B^{3}$ branched along the strings of a tangle $A$ (namely $P, Q$ or a reflection thereof) such that

(a) the numerator of $A$ is the unlink

(b) $A$ is not (freely) null; i.e., not pair diffeomorphic to the null tangle (Figure 1(b))

(that the 2-fold covering of $B^{3}$ branched over the tangle of Figure 4(a) is irreducible with incompressible boundary has been established in \$2).

LEMmA (A). If the numerator of any tangle $P$ is the unlink, then its branched cover $\tilde{B}_{P}^{3}$ is irreducible.

Proof. First observe that the 2-fold covering of $S^{3}$ branched over the 2-component unlink $P_{N}$ is $S^{2} \times S^{1}$ and that any 2-sphere embedded in $S^{2} \times S^{1}$ is, up to isotopy, either $S^{2} \times$ \{point $\}$ or the boundary of a 3-ball. 
Now $\tilde{B}_{P}^{3}$, the 2-fold covering of $B_{P}^{3}$ (the containing 3-ball) branched over $P$, is the complement in $S^{2} \times S^{1}$ of the interior of a solid torus $T$; namely, the 2-fold covering of the complementary 3-ball, $S^{3}-B_{P}^{3}$, branched over the complementary (null) tangle $P_{N}-P$, as illustrated in Figures 6(a) and (b) for the tangle $P$ of Figure 2(a).

We claim that $T$ is homologically essential in $S^{2} \times S^{1}$. To see this, first note that its core $c$ is the full preimage of an arc $b$ joining the two components of the unlink $P_{N}$, as indicated in Figures 6(a) and (b), and imagine a 2 -sphere $\Sigma$ which separates the components of $P_{N}$ and is met transversely by the arc $b$ (necessarily) an odd number of times, as in Figure 6(c). But then $\Sigma$ lifts to a pair of 2 -spheres $\Sigma_{+}$and $\Sigma_{-}$, each isotopic to $S^{2} \times$ \{point , and it follows readily that the intersection of $c \cdot \Sigma_{ \pm}$is odd and thus that $c$ is homologically essential $\bmod 2$.

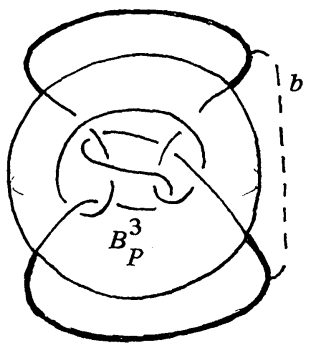

(a)

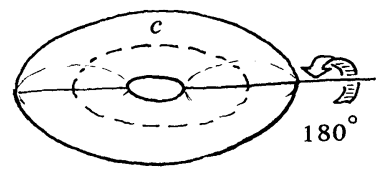

Branched covering of exterior of $B_{P}^{3}$

(b)

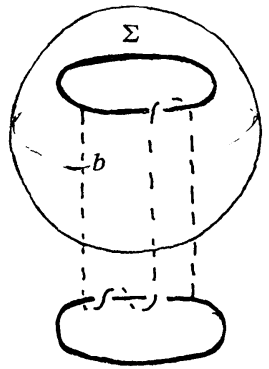

(c)

FIGURE 6

Finally, suppose $S$ is a 2-sphere in $\tilde{B}_{P}^{3}=S^{2} \times S^{1}$-int $T$. Now $T$ can't lie in the ball which $S$ bounds in $S^{2} \times S^{1}$, for then it would be homologically inessential in $S^{2} \times S^{1}$; thus $T$ lies outside the ball and it follows that $B_{P}^{3}$ is irreducible.

The following lemma is conceptually helpful although not strictly necessary here. It was perhaps first proved by $F$. Bonahon as an unstated corollary of his study of $\mathbf{Z}_{2}$-characteristic submanifolds [4]; the following proof has different scope.

Lemma (B). A tangle $P$ is freely null $\Leftrightarrow \tilde{B}_{P}^{3}$ is a solid torus.

Proof. Implication $\Rightarrow$ being obvious, suppose that the 2-fold branched cover $\tilde{B}_{P}^{3}$ is a solid torus. There exists, we claim, a meridianal disc $D$ of this solid torus whose image in $B^{3}$ is a disc $q(D)$ which is disjoint from $P$ and (hence) separates the two strings of $P$. Indeed, the $\mathbf{Z}_{2}$-equivariant Dehn's lemma-loop theorem, proved in [14], [10] and [17] (the last proof 
using minimal surface theory and valid for $\mathbf{Z}_{n}$ as well as $\mathbf{Z}_{2}$ ), assures a meridianal disc so that $q(D)$ is a disc transverse to $P$; but a transverse intersection of $\geq 1$ point with $P$ would make the disc $D$ a 2 -fold branched covering of $q(D)$, that separates $\tilde{B}_{P}^{3}$ because $q(D)$ separates $B^{3}$; this would prevent $D$ from being a meridianal disc.

Cutting $B^{3}$ apart along $q(D)$, we get two 3-balls $B_{+}^{3}$ and $B_{-}^{3}$, each containing one arc of $P$, say $P_{+}$and $P_{-}$respectively, and each covered by a 3-ball in the solid torus $\tilde{B}_{P}^{3}$. The $\mathbf{Z}_{2}$ Smith Conjecture proof of Waldhausen [23] (see [2] for the $\mathbf{Z} n$ Smith Conjecture proof) then shows that $P_{ \pm}$is unknotted in $B_{ \pm}^{3}$. Gluing $B^{3}$ back together we see that $P$ was freely null.

We pause to note that this argument, with at worst an induction on the number of existing strings, proves the following.

Proposition. Let $P$ be an oriented 1-submanifold of the 3-ball $B^{3}$ (a generalized free tangle) and let $n \geq 2$ be an integer. Then the $n$-fold cyclic covering $\tilde{B}_{P}^{3}$ of $B^{3}$ branched along $P$ is a solid multiple torus if and only if $P$ is freely null; i.e. isotopic to linear segments (secants). Also $\tilde{B}_{P}^{3}$ has compressible boundary if and only if there exists an embedded disc $q(D)$ in $B^{3}$ such that either

(i) $q(D)$ is disjoint from $P$ and separates $P$, or

(ii) $q(D)$ meets $P$ in one point $x$, transversely, and neither of the two (generalized) tangles into which $q(D)$ cuts $P$ is just an unknotted arc.

Returning to the proof that $M_{K}^{3}$ is irreducible, we have to check that, when a tangle $A$ satisfies conditions (a) and (b) preceeding the lemmas, then $\tilde{B}_{A}^{3}$ has incompressible boundary torus. This is true since cutting along a compression disc shows that any irreducible 3-manifold with compressible torus boundary is a solid torus, while $\tilde{B}_{A}^{3}$ is forbidden to be a solid torus by (b) and Lemma (B). In a specific case, e.g. Figure 4(a) or (b), it may be otherwise obvious that $\tilde{B}_{A}^{3}$ is not a solid torus.

\section{REFERENCES}

1. J. W. Alexander, Topological invariants of knots and links, Trans. Amer. Math. Soc., 30 (1928), 275-306.

2. H. Bass, J. Birman and J. Morgan, Editors, Columbia University Smith Conjecture Conference Proceedings, April 1979.

3. J. Birman, Orientation-reversing involutions on 3-manifolds, preprint.

4. F. Bonahon, Thèse de 3ième cycle, Orsay, 1979.

5. F. Bonahon and L. Siebenmann, Noeuds algebriques, (à paraitre). 
6. J. H. Conway, An enumeration of knots and links, and some of their algebraic properties, Computational Problems in Abstract Algebra, Pergamon Press, 1970, 329-358.

7. Skein theory, (to appear).

8. R. H. Fox, A quick trip through knot theory, Topology of 3-manifolds and Related Topics, Prentice Hall, 1962, 120-167.

9. D. E. Galewski and R. J. Stern, Orientation-reversing involutions on homology 3-spheres, Math. Proc. Cambridge Phil. Soc., 85 (1979), 449-451.

10. C. McA. Gordon and R. Litherland, On the Smith conjecture for homotopy 3-spheres.

11. W. Haken, Ein Verfahren zur Aufspaltung einer 3-Mannigfaltigkeit in irreduzible 3-Mannigfaltigkeiten, Math. Z., 76 (1961), 427-467.

12. W. C. Hsiang and P. S. Pao, Orientation-reversing involutions on homology 3-spheres.

13. K. Johannson, Lect. Notes in Math., No. 761, Springer, 1979 (to appear).

14. P. Kim and J. Tollefson, Splitting the PL involutions on nonprime 3-manifolds, Michigan Math. J., 27 (1980), 259-274.

15. S. Kinoshita and H. Terasaka, On unions of knots, Osaka Math. J., 9 (1957), 131-153.

16. J. Levine, A characterization of knot polynomials, Topology, 4 (1965), 135-141.

17. W. H. Meeks III and S. Yau, Topology of 3-dimensional manifolds and the embedding problem in minimal surface theory.

18. J. Montesinos, Revêtements doubles ramifiés, espaces fibrés de Seifert et seindements de Heegard, réduction de conférence à Orsay 1976, (à paraitre dans Astérisque).

19. K. Reidemeister, Knoten und Verkettungen, Math. Zeit., 29 (1929), 713-729.

20. D. Rolfsen, Knots and Links, Mathematical Lecture Series 7, Publish or Perish Inc., Berkeley, CA, 1976.

21. L. Siebenmann, Une introduction aux espaces fibres de Seifert, (à paraitre avec J. Montesinos).

22. J. M. Van Buskirk, Determinants of strongly positive amphicheiral knots are squares.

23. F. Waldhausen, Über involutionen der 3-Sphäre, Topology, 8 (1969), 81-91.

24. __ Eine Klasse von 3-dimensionalen Mannigfaltigkeiten I, II, Invent. Math., 3,4 (1967), 308-333, 87-117.

Received January 4, 1980 and in revised form September 12, 1980.

Université de Paris Sud

91405 ORSAY

FRANCE

AND

UNIVERSITY OF OREGON

EUGENE, OR 97403 



\section{PACIFIC JOURNAL OF MATHEMATICS EDITORS}

DONALD BABBITT (Managing Editor)

University of California

Los Angeles, CA 90024

Hugo Rossi

University of Utah

Salt Lake City, UT 84112

C. C. Moore and Arthur Ogus

University of California

Berkeley, CA 94720
J. DugunduI

Department of Mathematics

University of Southern California

Los Angeles, CA 90089-1113

R. FinN and H. SAmelson

Stanford University

Stanford, CA 94305

\section{ASSOCIATE EDITORS}
R. ARENS
E. F. BECKENBACH
B. H. NeUmaNN
F. WolF
K. YoshidA (1906-1982)

\section{SUPPORTING INSTITUTIONS}

UNIVERSITY OF ARIZONA

UNIVERSITY OF BRITISH COLUMBIA

CALIFORNIA INSTITUTE OF TECHNOLOGY

UNIVERSITY OF CALIFORNIA

MONTANA STATE UNIVERSITY

UNIVERSITY OF NEVADA, RENO

NEW MEXICO STATE UNIVERSITY

OREGON STATE UNIVERSITY
UNIVERSITY OF OREGON

UNIVERSITY OF SOUTHERN CALIFORNIA

STANFORD UNIVERSITY

UNIVERSITY OF HAWAII

UNIVERSITY OF TOKYO

UNIVERSITY OF UTAH

WASHINGTON STATE UNIVERSITY

UNIVERSITY OF WASHINGTON 


\section{Pacific Journal of Mathematics}

\section{Vol. 106, No. $1 \quad$ November, 1983}

John Ballard, Clifford's theorem for algebraic groups and Lie algebras . . . . . 1

Philip Richard Bartick, II and Edwin Duda, Finite-to-one open mappings

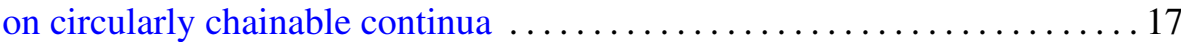

Frank Hayne Beatrous, Jr., $H^{\infty}$-interpolation from a subset of the

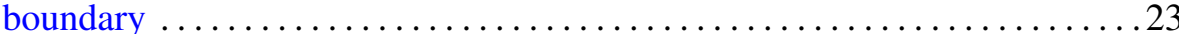

Sterling K. Berberian, Expectations in semifinite algebras ............ 33

Heron S. Collins and Wolfgang Ruess, Weak compactness in spaces of compact operators and of vector-valued functions $\ldots \ldots \ldots \ldots \ldots \ldots . \ldots 45$

David Downing and William O. Ray, Renorming and the theory of

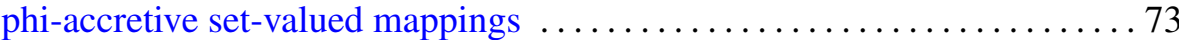

Harvey Charles Greenwald, On the theory of homogeneous Lipschitz

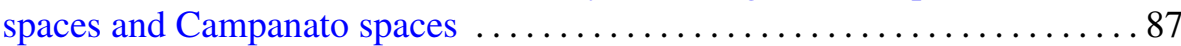

Irwen Valle Guadalupe and Lucio Ladislao Rodriguez, Normal curvature

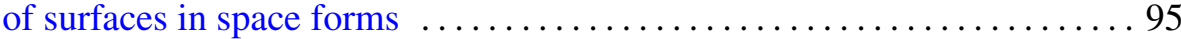

Boju Jiang, On the computations of the Nielsen number ............... 105

William H. Julian, Ray Mines, III and Fred Richman, Alexander

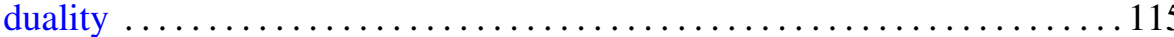

Midori Kobayashi, The connected component of the idèle class group of an

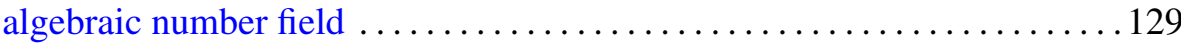

Ib Henning Madsen, Charles B. Thomas and C. Terence C. (Charles)

(Clegg) Wall, Topological spherical space form problem. III.

Dimensional bounds and smoothing

A. Sapounakis, The existence of strong liftings for totally ordered measure

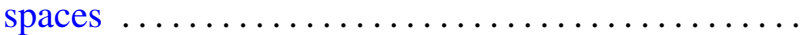

Gary Seitz, The root subgroups for maximal tori in finite groups of Lie type

Laurent Siebenmann and James M. Van Buskirk, Construction of irreducible homology 3-spheres with orientation reversing involution 